\title{
Tolerance of Nectria haematococca MP VI to the Phytoalexin Pisatin in the Absence of Detoxification
}

\author{
By TIMOTHY P. DENNY† AND HANS D. VANETTEN* \\ Department of Plant Pathology, 334 Plant Science Building, Cornell University, \\ Ithaca, New York 14853-0331, U.S.A.
}

(Received 8 February 1983)

\begin{abstract}
Most isolates of Nectria haematococca mating population (MP) VI detoxify pisatin, the major phytoalexin produced by pea. However, there is evidence of another pisatin tolerance mechanism that does not depend on pisatin degradation. To facilitate further studies on nondegradative tolerance, a bioassay was developed in which culture turbidity was used as a measure of mycelial growth. Under conditions of this assay, four $N$. haematococca MP VI isolates degraded $0.4 \mathrm{mM}$-pisatin within $15 \mathrm{~h}$, but two other isolates required more than $24 \mathrm{~h}$. A remaining group of four isolates did not degrade pisatin. These three groups were typified by the isolates 126-71, T-77 and 126-80, respectively. Regardless of their ability to degrade pisatin, however, all 10 isolates grew equally well after addition of $0.4 \mathrm{~mm}$-pisatin, suggesting that detoxification may not be needed for pisatin tolerance during the short time interval of this assay. The existence of a nondegradative tolerance in isolate 126-71 was confirmed when additional experiments showed that growth could occur (i) before degradation had reduced pisatin below initially noninhibitory levels, and (ii) when pisatin degradation was inhibited by $2 \%$ ethanol. When tested in growth medium, pretreatment with a noninhibitory concentration of pisatin for $3 \mathrm{~h}$ enhanced equally the ability of isolates 126-71 and 126-80 to tolerate pisatin. After cultures were pretreated with pisatin while temporarily suspended in buffer, isolates 12671 and T-77 were stimulated to degrade pisatin, and they grew better than did isolate 126-80. Again, however, growth began before there was significant detoxification, especially for isolate $\mathrm{T}-77$. Thus, these three isolates all appeared to have an inducible, nondegradative tolerance to pisatin. Nectria haematococca MP I isolate T-145 lacked an adaptive tolerance to pisatin and was more sensitive to pisatin than any of the MP VI isolates.
\end{abstract}

\section{INTRODUCTION}

Phytoalexins are antimicrobial plant metabolites that accumulate in plant tissue in response to the presence of micro-organisms (Bailey \& Mansfield, 1982). However, the growth in vitro of some plant pathogenic fungi is relatively uninhibited by concentrations of their hosts' phytoalexins that are inhibitory to many fungi (Cruickshank, 1962; Cruickshank \& Perrin, 1971; VanEtten, 1973). The ability of a plant pathogen to tolerate its host's phytoalexins is thought to be one factor required for pathogenicity in some host-parasite combinations (Cruickshank, 1965; Bailey \& Mansfield, 1982). Evidence for the importance of phytoalexin tolerance in pathogenicity has been provided by studies with Nectria haematococca Berk. and $\mathrm{Br}$. mating population (MP) VI (imperfect stage: Fusarium solani). Within a collection of MP VI field isolates the natural variation in virulence on pea correlated with tolerance to pisatin, the primary pea phytoalexin; only the most tolerant isolates were highly virulent (VanEtten et al., 1980). Furthermore, when crosses were made using parents that differ in both of these traits none of the segregating progeny that were most sensitive to pisatin were even moderately virulent (Tegtmeier \& VanEtten, 1982).

$\dagger$ Present address: Department of Pediatrics, Division of Infectious Diseases, University of North Carolina, Chapel Hill; North Carolina 27514, U.S.A.

Abbreviation: GA medium, glucose-asparagine medium. 
One mechanism of phytoalexin tolerance in $N$. haematococca MP VI and some other plant pathogenic fungi is thought to involve the ability to degrade phytoalexins to less inhibitory compounds (Harborne \& Ingham, 1978; VanEtten et al., 1982). However, there is evidence that another mode of phytoalexin tolerance, of unknown biochemical basis, can exist independently of phytoalexin detoxification (Denny \& VanEtten, 1981; Higgins, 1978; Kistler \& VanEtten, 1981; Skipp \& Carter, 1978; VanEtten \& Stein, 1978). Nondegradative tolerance to phytoalexins needs to be more thoroughly characterized, because it may be a prerequisite for subsequent phytoalexin detoxification (Denny \& VanEtten, 1981; Rossall \& Mansfield, 1978).

Most of the previously employed bioassays (e.g., growth for days on semi-solid medium) are ill-suited for observing nondegradative phytoalexin tolerance. Rapid, transient changes in growth may be overlooked, and when detoxification occurs, phytoalexin quantification is difficult (Bailey et al., 1976; Skipp \& Bailey, 1977). The best demonstrations of nondegradative tolerance have used microscopic observations of spores or mycelium growing in liquid medium (Denny \& VanEtten, 1981; Higgins, 1978). The use of liquid culture systems has the advantages that (i) initial effects of phytoalexins (or other compounds) can be observed immediately after their addition, and (ii) localized depletion of phytoalexins is avoided, thus simplifying quantification (Skipp \& Bailey, 1977). Unfortunately, microscopic assays are cumbersome and laborious, and experiments with them have consequently been limited in number and design. By adapting the technique described by Trinci (1972), in which turbidity of a filamentous fungus growing in liquid culture is used as a measure of mycelial growth, a convenient and flexible assay was developed that made a more intensive study of nondegradative tolerance practical.

Isolates of $N$. haematococca MP VI known to differ in their ability to detoxify pisatin were reexamined using the turbidimetric assay. The results suggest that an adaptive, nondegradative tolerance to pisatin facilitates growth during the period shortly after exposure to the phytoalexin, regardless of an isolate's degradative ability.

\section{METHODS}

Fungal isolates and media. Nectria haematococca MP VI isolates were those that had been used in previous studies (Denny \& VanEtten, 1982; Tegtmeier \& VanEtten, 1982; VanEtten et al., 1980). The field isolates T-23, T63 and T-77, and ascospore isolates 6-36, 126-47 and 126-71 are capable of detoxifying pisatin. Field isolate T-213 and ascospore isolates 126-2, 126-11 and 126-80 lack the ability to degrade pisatin. Nectria haematococca MP I isolate T-145 was used for comparison with the MP VI isolates; T-145 does not detoxify pisatin (Denny \& VanEtten, 1982) and is known to be more sensitive to pisatin than any MP VI isolates (VanEtten et al., 1980).

Stock cultures of the fungi were maintained on V-8 juice agar slants as previously described (Denny \& VanEtten, 1981). Conidia from the stocks were transferred to fresh V-8 slants 1 to 2 weeks before each experiment. Glucose-asparagine (GA) liquid medium (VanEtten \& Stein, 1978) containing 3\% (w/v) glucose was used for all shake cultures.

Fungal growth bioassay. A mycelial suspension of each isolate was obtained by inoculating $300 \mathrm{ml}$ flasks with $100 \mathrm{ml} \mathrm{GA}$ medium containing $2 \times 10^{5}$ macroconidia $\mathrm{ml}^{-1}$ and incubating the flasks at $28 \pm 1{ }^{\circ} \mathrm{C}$ on an orbital shaker (125 r.p.m.) for 12 to 14 h. After this incubation period, the mycelial suspensions were decanted away from the ring of mycelium that formed on the walls of the flasks and any remaining clumps of mycelium were removed with a sterile pipette. Growth measurements on these mycelial suspensions were performed in $250 \mathrm{ml}$ culture flasks fitted with a standard $18 \mathrm{~mm}$ (outer diameter) culture tube attached as a side arm (a Nephelo culture flask). Each culture flask received 6 to $12 \mathrm{ml}$ of the mycelial suspension and the flasks were incubated as above. Turbidity of the mycelial suspensions was determined at approximately hourly intervals by pouring the suspensions into the sidearms and reading the absorbance on a Bausch \& Lomb Spectronic 20 set at $620 \mathrm{~nm}$, using water as a blank. Dry weight of the mycelium was determined for aliquots of the suspensions by collecting the mycelium on tared glass fibre filters (Whatman GF/A; $2 \cdot 4 \mathrm{~cm}$ ) and drying at $70^{\circ} \mathrm{C}$ for at least $24 \mathrm{~h}$.

The initial absorbances of the mycelial suspensions were between 0.2 and 0.5 units. When the initial absorbance readings within a set of experiments fell within a $0 \cdot 1$ unit range the readings were subsequently normalized to a common figure, and the results were reported as averages of this normalized data. When initial readings were outside of this range, the results of a representative experiment are given.

Pisatin (6a-hydroxy-3-methoxy-8,9-methylenedioxy pterocarpan), purified from pea by published methods (VanEtten et al., 1975) was added to cultures in an appropriate volume of DMSO to give a final concentration of $2 \%(\mathrm{v} / \mathrm{v})$ DMSO. Controls received $2 \%$ DMSO, which reduced the rate of growth by less than $2.5 \%$.

Recovery and quantification of pisatin. The first step in pisatin detoxification is a 3-O-demethylation (VanEtten $e t$ al., 1975). Unlike pisatin, the metabolic products containing the methyl moiety removed from pisatin during the 
reaction are not extracted from an aqueous phase into toluene. One method of quantifying pisatin exploited this difference in solubility by using pisatin that was specifically labelled with ${ }^{14} \mathrm{C}$ in the $3-O$-methyl position (VanEtten et al., 1980). The extent of demethylation was determined by shaking a $0.5 \mathrm{ml}$ aliquot of treated mycelial suspension (pisatin specific activity $\geqslant 1.4 \times 10^{4}$ d.p.m. $\mu \mathrm{mol}^{-1}$ ) together with $4.5 \mathrm{ml}$ scintillation fluid (1 litre toluene, $5 \cdot 5 \mathrm{~g}$ 2,5-diphenyloxazole) in a $(7 \mathrm{ml})$ minivial. The amount of ${ }^{14} \mathrm{C}$ extracted into the scintillation fluid was measured with a liquid scintillation spectrometer. The $\left[{ }^{14} \mathrm{C}\right]$ methyl metabolites produced during degradation remained in the aqueous phase and contributed little to the total ${ }^{14} \mathrm{C}$ detected.

In several experiments, pisatin degradation was monitored by extracting unlabelled pisatin from treated cultures with chloroform $(1: 1, \mathrm{v} / \mathrm{v})$. The cultures were extracted three times, and then the chloroform extracts were pooled, reduced in volume and applied to silica gel TLC plates (Analtech; GF). The plates were developed in chloroform/methanol $(25: 1, \mathrm{v} / \mathrm{v})$, and the area of silica adjacent to a co-migrating pisatin standard was removed from the plate and eluted with excess ethanol. Pisatin was identified by its UV spectrum and by its characteristic acid-catalysed dehydration product, anhydropisatin (3-methoxy-8,9-methylenedioxy pterocarp-6a-ene) (Perrin \& Bottomley, 1962). Pisatin was quantified by using its molar absorption coefficient $(\varepsilon)$ in neutral ethanol $(\log \varepsilon=3.86$ at $309 \mathrm{~nm})$ (Perrin \& Bottomley, 1962).

Preliminary experiments showed that, for isolates 126-71 and 126-80, the two methods of quantifying pisatin gave the same results. Thereafter, pisatin degradation was monitored only with the ${ }^{14} \mathrm{C}$ assay.

\section{RESULTS}

\section{Correlation of dry weight with turbidity}

If turbidity is to be a useful and reliable measurement of fungal growth, then absorbance must be related to dry weight in a straightforward manner. At the end of the initial 12 to $14 \mathrm{~h}$ period, there were no mycelial pellets in the homogeneous suspension, and hyphae growing from the macroconidia were 500 to $700 \mu \mathrm{m}$ long and just beginning to branch. Mycelial dry weight of each isolate increased exponentially throughout the assay, with a dcabling time of about $3 \mathrm{~h}$; stationary growth phase did not begin until dry weight was greater than $8 \mathrm{mg} \mathrm{ml}^{-1}$. Increasing turbidity was closely correlated with increasing dry weight (Fig. 1), but the relationship was not constant over the full range of these variables. For dry weights of less than about $0.3 \mathrm{mg} \mathrm{ml}^{-1}$, mycelial growth occurred with little change in turbidity. Therefore, assays were designed to run between an absorbance of $\mathbf{0 . 2}$ units, where turbidity became an increasingly sensitive indicator of changes in dry weight, and 1.2 units where increasing viscosity and spurious light-scatter made readings less accurate. In this range, turbidity was approximately linear with log (dry weight). The production of microconidia by some isolates had little effect on the turbidity of the cultures. All the isolates tested conformed to the same absorbance-to-dry-weight relationship (Fig. 1).

\section{Response to $0.4 \mathrm{~mm}$-pisatin}

Ten $N$. haematococca MP VI isolates were surveyed for their response to the addition of $0.4 \mathrm{~mm}$-pisatin to the culture medium. At intervals during the incubation, aliquots were removed to determine pisatin degradation. The isolates could be divided into three groups based on their ability to degrade pisatin: (i) rapid degradation (isolates 126-47, 126-71, T-23 and T-63); (ii) slow degradation (isolates 6-36 and T-77); and (iii) no degradation (isolates 126-2, 126-11, 126-80 and T-213). Growth and metabolism by single isolates representative of each group are illustrated in Fig. 2. After a period of initial sensitivity to pisatin during which growth was inhibited, all the isolates tested developed a tolerance to pisatin and growth resumed. That the isolates grew equally well regardless of their rate of pisatin degradation suggests that detoxification may not be needed for the expression of pisatin tolerance by any of the isolates for the duration of this assay.

\section{Expression of nondegradative tolerance in isolate 126-71}

Isolate 126-71 was used to test whether adaptation to pisatin and pisatin detoxification could be dissociated in an isolate that has the capacity to detoxify pisatin. In one experiment, the concentration of pisatin was increased to $0.525 \mathrm{~mm}$ or $0.6 \mathrm{~mm}$ and the effects on growth and pisatin detoxification examined (Fig. 3). At either concentration, growth resumed before the concentration of pisatin began to decrease at a rapid rate. In a related experiment, the 


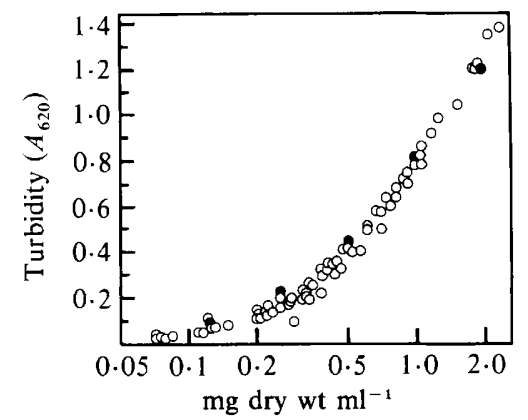

Fig. 1. Correlation of dry weight and turbidity for $N$. haematococca MP VI isolates. The 10 isolates listed in Methods and three additional $N$. haematococca MP VI isolates were grown in GA medium. Aliquots were removed from each culture at intervals of $3 \mathrm{~h}$. The absorbance at $620 \mathrm{~nm}$ was measured and the dry weights of the mycelium determined. The filled circles are the data for isolate 126-80.
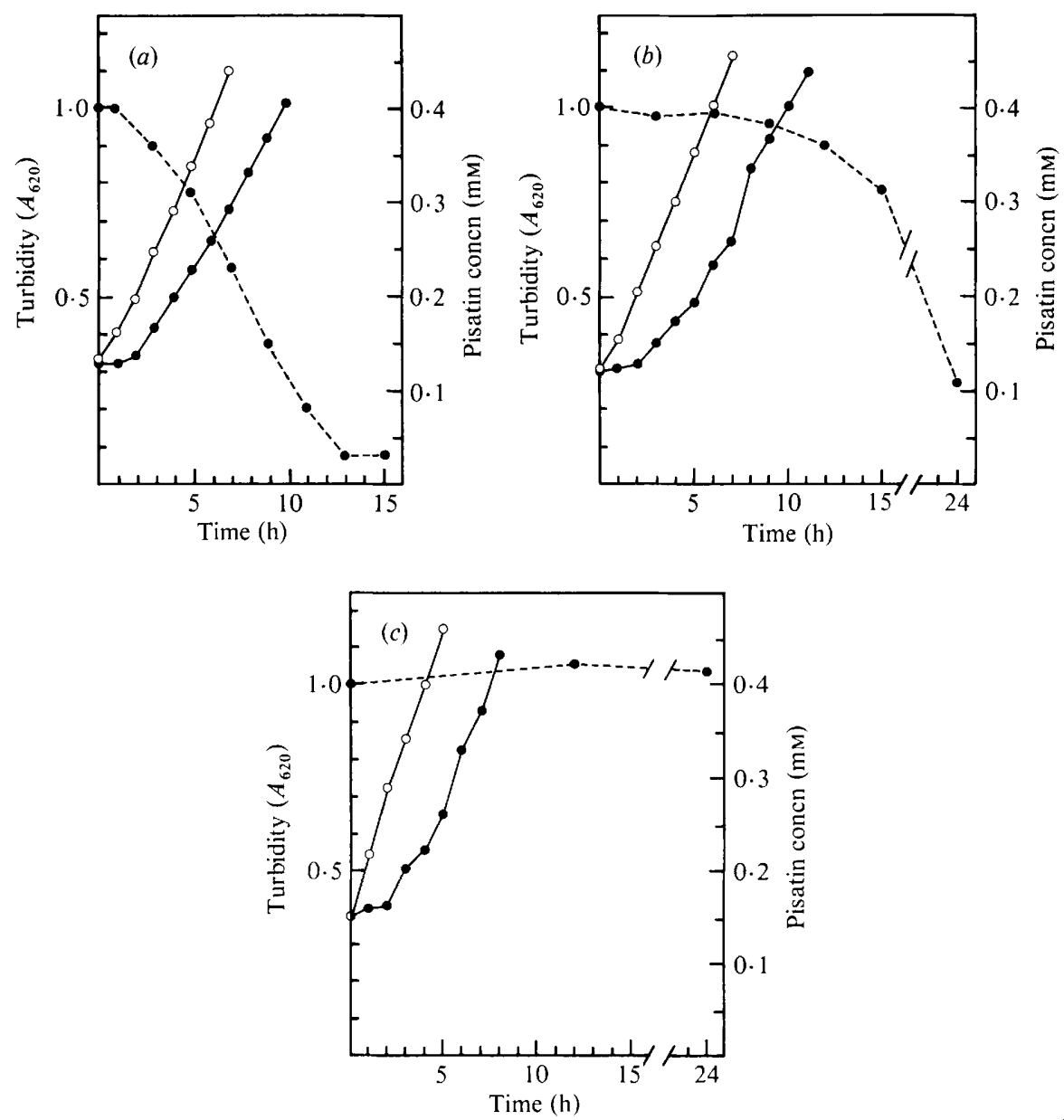

Fig. 2. Growth and degradation of pisatin by three $N$. haematococca MP VI isolates. Growth of mycelium in the $2 \%$ DMSO control $(O)$ or after treatment with $0.4 \mathrm{~mm}$-pisatin $(O)$ was determined turbidimetrically. After resuming growth in the pisatin-amended medium, all three isolates grew at about $73 \%$ of the rate of the control. The amount of pisatin remaining (dashed line) was determined by periodically assaying aliquots of the pisatin-treated cultures. Data are from typical experiments, representative of at least three experiments for each isolate. $(a)$ Isolate $126-71,(b)$ isolate T-77, and $(c)$ isolate $126-80$. 


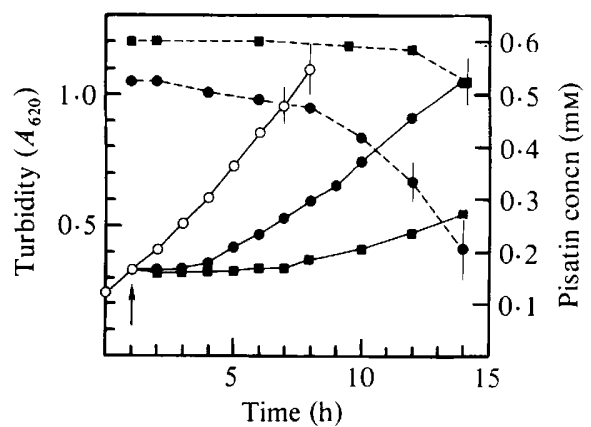

Fig. 3. Growth and degradation of pisatin by $N$. haematococca MP VI isolate 126-71. At the time designated by the arrow, cultures received either $2 \%$ DMSO $(O), 0.525 \mathrm{~mm}$-pisatin $(O)$, or $0.6 \mathrm{~mm}$ pisatin ( $\square$ ). Pisatin degradation (dashed lines) was determined simultaneously as in Fig. 2. Data are the means of two experiments. Error bars are shown only for those points where the S.E.M. exceeded 0.03 units.

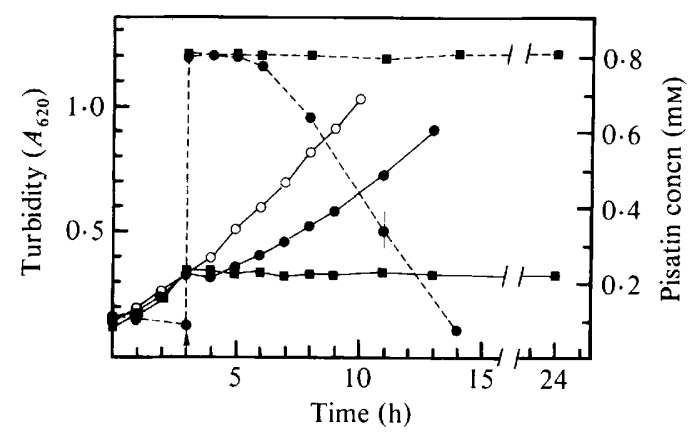

Fig. 4. Effects of pisatin pretreatment on growth and pisatin degradation by $N$. haematococca MP VI isolate 126-71. One culture $(O)$ received $0.1 \mathrm{~mm}$-pisatin at $0 \mathrm{~h}$ and an additional $0.7 \mathrm{~mm}$-pisatin at $3 \mathrm{~h}$ (arrow). A paired culture ( $)$ was only treated at $3 \mathrm{~h}$ with $0.8 \mathrm{~mm}$-pisatin. Pisatin degradation (dashed lines) was determined simultaneously as in Fig. 2. The control $(O)$ received $2 \%$ DMSO. Data are the means of two experiments; S.E.M. is shown where it exceeded 0.025 units.

concentration of pisatin was increased to $0.8 \mathrm{~mm}$ (Fig. 4), an amount that completely inhibited isolate 126-71 if the compound was added all at once. However, if the pisatin was added in increments, first to a concentration of $0.1 \mathrm{mM}$ for $3 \mathrm{~h}$ and then raised to $0.8 \mathrm{mM}$, isolate 126-71 grew and degraded the phytoalexin. The $3 \mathrm{~h}$ pretreatment induced an enhanced tolerance of pisatin, and isolate 126-71 began to grow even though the pisatin concentration was above the $0.6 \mathrm{~mm}$ that inhibited the growth of non-adapted mycelium for $7 \mathrm{~h}$ (Fig. 3). Isolate 126-80, which is unable to degrade pisatin, was similarly induced to tolerate $0.8 \mathrm{~mm}$-pisatin using the same protocol, clearly illustrating that adaptation to pisatin does not require pisatin detoxification. In another experiment, pisatin dissolved either in ethanol or in DMSO was added to identical cultures of isolate 126-71 (Fig. 5). Although growth was essentially the same in the two solvents, degradation of pisatin was much slower in ethanol than in DMSO. The reason for this solvent effect was not determined, but it again illustrates that pisatin detoxification is not essential for adaptation to pisatin.

\section{An attempt to demonstrate that detoxification enhances growth}

When $N$. haematococca MP VI isolates are grown for 3 to $4 \mathrm{~d}$ on semi-solid medium that contains pisatin and DMSO (i.e., a radial growth bioassay) those isolates that detoxify pisatin are significantly less inhibited than those that do not (Denny \& VanEtten, 1982; Tegtmeier \& VanEtten, 1982; VanEtten et al., 1980). In two experiments using published procedures for the 


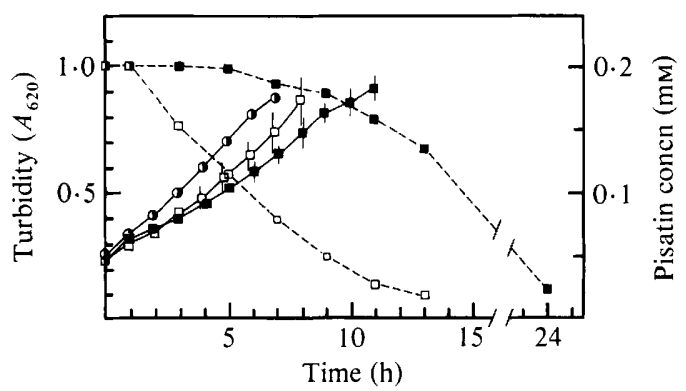

Fig. 5. The effect of carrier solvent on growth and degradation of pisatin by $N$. haematococca MP VI isolate 126-71. Pisatin (0.2 mM) was added in either DMSO ( $\square$ ) or ethanol ( $\square$ ) at a final concentration of $2 \%$ solvent. Controls received only the solvents at $2 \%(\mathbf{O})$. Growth (solid lines) and degradation (dashed lines) were determined simultaneously as in Fig. 2. Data are the means of two experiments; S.E.M. for absorbance is reported as for Fig. 3; S.E.M. for degradation did not exceed $0.01 \mathrm{mM}$.
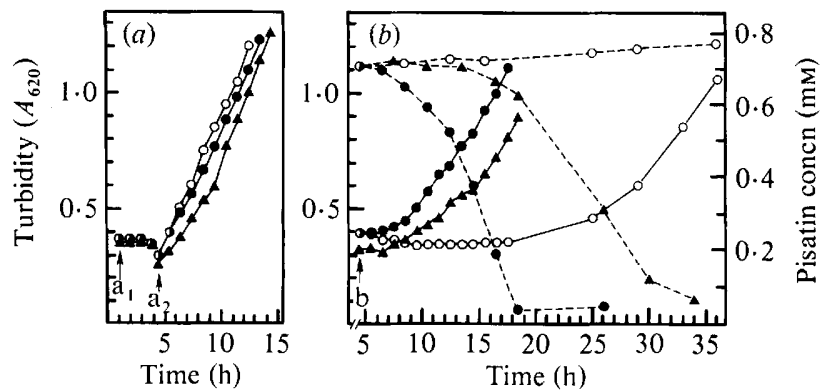

Fig. 6. Induction of pisatin tolerance in $N$. haematococca MP VI by pretreatment with pisatin. (a) The mycelium for cultures of 126-80 (O), 126-71 (O) and T-77 (A) was harvested on glass fibre filters, washed with $50 \mathrm{~mm}$-potassium phosphate buffer $(\mathrm{pH} \mathrm{6.0)}$ and resuspended in buffer to the original volume. The suspensions were incubated at $28^{\circ} \mathrm{C}$ for $1 \mathrm{~h}$ and then treated with $0 \cdot 1 \mathrm{~mm}$-pisatin (arrow $\mathrm{a}_{1}$ ). Incubation continued for a further $3 \mathrm{~h}$ before the mycelium was harvested, washed, resuspended in GA medium and the culture of each isolate divided into two aliquots. One aliquot of each isolate was treated with $2 \%$ DMSO (arrow $a_{2}$ ). (b) The other aliquots received 0.7 mM-pisatin (arrow b). Growth (solid lines) and pisatin degradation (dashed lines) were determined simultaneously as in Fig. 2. The symbols are as in $(a)$. Results are from a typical experiment, representative of three experiments.

radial growth bioassay (Denny \& VanEtten, 1981; VanEtten et al., 1980), isolates $126-71$ and T77 (which detoxify pisatin) were inhibited an average of $2.5 \%$ and $11.5 \%$, respectively, by $0.5 \mathrm{~mm}$-pisatin when compared to the rate of growth of controls. Isolate $126-80$ does not degrade pisatin and was inhibited, on average, by $62 \%$. Pisatin detoxification appears to be important for tolerance during such extended periods of growth (Tegtmeier \& VanEtten, 1982). However, such large differences in pisatin tolerance were not observed during the turbidimetric assay, possibly because the short duration of this assay did not allow the potential advantages of detoxification to be fully expressed. If the ability to detoxify pisatin could be induced in isolates 126-71 and T-77 without inducing nondegradative tolerance, then these isolates should degrade pisatin substantially before beginning to grow and then grow more rapidly than isolate $126-80$ treated similarly. Such an experiment seemed feasible because, when mycelium was temporarily suspended in buffer, the ability to detoxify pisatin was known to be highly inducible (VanEtten \& Barz, 1981), whereas preliminary experiments indicated that, at least for isolate $126-80$, these same conditions were unfavourable for the induction of nondegradative pisatin tolerance. The details of such an experiment are given in Fig. 6.

None of the isolates tested grew in or detoxified $0.7 \mathrm{~mm}$-pisatin if they were pretreated in buffer for $3 \mathrm{~h}$ with $2 \%$ DMSO instead of pisatin. Pretreatment of mycelium in buffer with pisatin induced nondegradative tolerance in isolate 126-80, but the adaptation was slow to be expressed. In contrast, the tolerance expressed by isolates 126-71 and T-77 developed much 


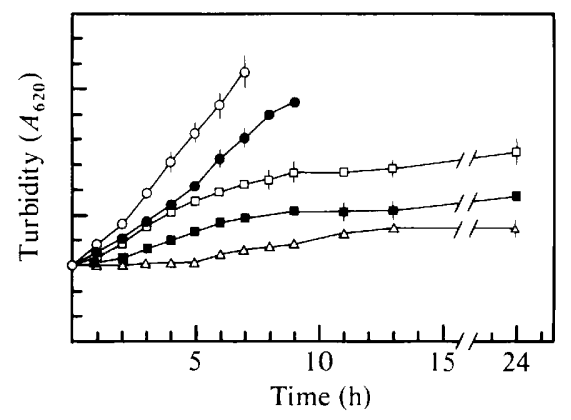

Fig. 7. The effect of pisatin on $N$. haematococca MP I isolate T-145. Cultures received either $0 \cdot 1 \mathrm{mM}$ $(\bigcirc), 0.2 \mathrm{~mm}(\square), 0.3 \mathrm{~mm}(\square)$ or $0.4 \mathrm{~mm}(\triangle)$ pisatin. The control $(O)$ was treated with $2 \%$ DMSO. There was no degradation of pisatin during the assay. Data are the means of three or more experiments; S.E.M. is reported as for Fig. 3.

more rapidly. The ability to detoxify pisatin was also induced in isolates 126-71 and T-77. However, these isolates did not degrade pisatin to concentrations that were initially noninhibitory (less than $0.4 \mathrm{~mm}$ ) before beginning to grow. Instead, as in the experiments above, growth began (especially for T-77) before there was significant pisatin detoxification, again suggesting the induction of a nondegradative tolerance. The differences in tolerance exhibited by these isolates during this experiment can be explained by differences in the inducibility of their nondegradative tolerance. The experiment failed to demonstrate clearly the effect of detoxification.

\section{Effects of pisatin on a N. haematococca MP I isolate}

Unlike the MP VI isolates, $N$. haematococca MP I isolate T-145 was inhibited by relatively low concentrations of pisatin, but only after an initial period of moderate growth (Fig. 7). Attempts to induce an enhanced tolerance of pisatin in T-145 by pretreating for $3 \mathrm{~h}$ with $0 \cdot 1 \mathrm{~mm}$-pisatin failed; the effects of incremental treatments were additive.

\section{DISCUSSION}

The principle of using light-scattering to measure indirectly the growth of unicellular organisms is well known (Mallette, 1969). Although less well known, under the proper conditions the same principle appears to be applicable to fungi (Alberghina et al., 1979; Trinci, 1972) and actinomycetes (Flowers \& Williams, 1977). When using multicellular organisms, success is critically dependent on achieving a homogeneous suspension without clumps. With a suitable combination of medium, culture conditions and size of inoculum, all but a few isolates of $N$. haematococca that were tested had acceptable growth habits. In addition, a reliable relationship between turbidimetric and gravimetric measurements was established (Fig. 1).

In earlier work with $N$. haematococca MP VI (VanEtten \& Barz, 1981; Wit-Elshove \& Fuchs, 1971), glucose was found to inhibit the degradation of pisatin. In contrast, during the turbidimetric assay those isolates capable of degrading pisatin did so in spite of a $3 \%$ glucose concentration. Furthermore, degradation was only slightly enhanced if isolate 126-71 was treated while in buffer instead of in GA medium. However, cultures grown for $24 \mathrm{~h}$ in GA medium degraded substantially less pisatin than did cultures grown for $12 \mathrm{~h}$ (unpublished observations), which suggests that the catabolite repression seen by other researchers could be attributed partially to their using older cultures.

The turbidimetric assay revealed an immediate, transient cessation of growth of $N$. haematococca MP VI isolates during the initial period of contact with pisatin (Figs 2, 3 and 4). Inhibition by a toxicant implies that the compound has contacted a sensitive site on or within the organism. That fungi which would be classified as tolerant in more common bioassays are initially sensitive to phytoalexins has been most easily detected during microscopic observations 
(Denny \& VanEtten, 1981; Higgins, 1978; Smith, 1976; Sprang \& VanEtten, 1978). Detection of the short period of complete inhibition using the turbidimetric measurement of growth is a good demonstration of the assay's sensitivity. After the period of inhibition, MP VI isolates adapted and resumed growth when pisatin concentrations were less than about $0.6 \mathrm{~mm}$. Higher concentrations were almost completely inhibitory.

The limited difference in pisatin tolerance among the isolates differing in their ability to degrade pisatin was unexpected. Since the radial growth assay (Denny \& VanEtten, 1981; VanEtten et al., 1980) used previously and the turbidimetric assay are so dissimilar, it is not surprising that some disparities would be observed when making comparisons. Nevertheless, an observation of enhanced growth due to detoxification was expected. Two (unpublished) observations may explain why this was not observed. First, when determining pisatin's inhibitory effects with the radial growth assay, differences in growth rate among isolates that differ in their ability to detoxify pisatin were detectable only after the first $24 \mathrm{~h}$. The similarities in growth response observed for the different isolates in the turbidity assay may correspond to this early phase of the radial growth assay. Liquid cultures would enter stationary growth phase before the full effect of detoxification could be expressed. Second, during radial growth bioassays several of the isolates that cannot detoxify pisatin, including 126-80, produced larger amounts of aerial hyphae than did isolates that detoxify pisatin. Therefore, the total increase in cell mass is not determined as accurately by the radial growth bioassay as it is by the turbidimetric assay.

For those $N$. haematococca MP VI isolates with reduced ability to degrade pisatin (T-77 and 126-80) the adaptation observed during the turbidimetric assay was clearly the result of an induced tolerance to pisatin that does not require pisatin detoxification. These MP VI isolates were substantially more tolerant to pisatin than the non-detoxifying MP I isolate T-145. Without the ability to adapt to pisatin, isolate $\mathrm{T}-145$ was tolerant to only about $0 \cdot 1 \mathrm{~mm}$-pisatin. The slow cessation of growth of isolate T-145 in the presence of $0.2 \mathrm{~mm}$-pisatin implies either that an effective concentration of pisatin slowly accumulated at the active site or that the metabolic system inhibited at time zero was not immediately required for growth. Immediate inhibition by $0.4 \mathrm{mM}$-pisatin of both MP I and MP VI isolates could be the result of the phytoalexin acting at additional sensitive sites.

Several experiments demonstrated that $N$. haematococca MP VI isolate 126-71 has a nondegradative tolerance mechanism in addition to its ability to detoxify pisatin. Assuming that the tolerance of isolate 126-71 before induction by pisatin is similar to that of T-145, and that detoxification is the sole component of tolerance, growth of 126-71 would have resumed only after pisatin had been reduced to about $0 \cdot 1 \mathrm{~mm}$. Isolate 126-71 was able to grow in much higher concentrations of pisatin, either with or without previous exposure to pisatin. Additionally, regardless of its rate of detoxification (Fig. 5), 126-71 grew well in 0.2 mM-pisatin. The rapid expression of nondegradative pisatin tolerance is similar to earlier work with the phytoalexins medicarpin and maackiain (Denny \& VanEtten, 1981), and reinforces the concept that nondegradative tolerance may be a prerequisite for the development of detoxificative tolerance.

Whether the nondegradative pisatin tolerance observed for isolates 126-71, T-77 and 126-80 is the same for each isolate could not be determined. However, because the two ascospore isolates (126-71 and 126-80) have a similar genetic background, the tolerance is likely to be similar even though inducibility during starvation conditions appeared to differ (Fig. 6). If the 10 isolates studied are representative of $N$. haematococca MP VI then it is possible that all isolates of this mating population possess an inducible nondegradative pisatin tolerance. Therefore, a more intensive characterization of nondegradative phytoalexin tolerance using one isolate that cannot degrade pisatin may provide information applicable to all MP VI isolates.

This work was supported in part by Rockefeller Foundation Grant RF 79049-13 and USDA/SEA Grant 592361-0-1-477-0.

\section{REFERENCES}

Alberghina, A., Benni, A. \& Fantino, M. G. (1979). Effects of temperature and polyphenols on fusaria growth. Phytopathologische Zeitschrift 96, 40-49.
Bailey, J. A. \& Mansfield, J. W. (1982). Phytoalexins. Glasgow \& London: Blackie.

Bailey, J. A., Carter, G. A. \& Skipp, R. A. (1976). 
The use and interpretation of bioassays for fungitoxicity of phytoalexins in agar medium. Physiological Plant Pathology 8, 189-194.

Cruickshank, I. A. M. (1962). Studies on Phytoalexins. IV. The antimicrobial spectrum of pisatin. Australian Journal of Biological Sciences 15, 147-159.

Cruickshank, I. A. M. (1965). Phytoalexins in the Leguminosae with special reference to their selective toxicity. Tagungsberichte. Deutsche Akademie der Landwirtschaftswissenschaften zu Berlin 74, 313-332.

Cruickshank, I. A. M. \& Perrin, D. R. (1971). Studies on phytoalexins. XI. The induction, antimicrobial spectrum and chemical assay of phaseollin. Phytopathologische Zeitschrift 70, 209-229.

DenNY, T. P. \& VANETTEN, H. D. (1981). Tolerance by Nectria haematococca MP VI of the chickpea (Cicer arietinum) phytoalexins medicarpin and maackiain. Physiological Plant Pathology 19, 419-437.

DenNy, T. P. \& VANETTEN, H. D. (1982). Metabolism of the phytoalexins medicarpin and maackiain by Fusarium solani. Phytochemistry 21, 1023-1028.

Flowers, T. H. \& Williams, S. T. (1977). Measurement of growth rates of streptomycetes: comparison of turbidometric and gravimetric techniques. Journal of General Microbiology 98, 285-289.

HARBORNE, J. B. \& InGHAM, J. L. (1978). Biochemical aspects of the coevolution of higher plants with their fungal parasites. In Biochemical Aspects of Plant and Animal Coevolution, pp. 343-405. Edited by J. B. Harborne. New York: Academic Press.

HigGins, V. J. (1978). The effect of some pterocarpan phytoalexins on germ tube elongation of Stemphylium botryosum. Phytopathology 68, 339-345.

Kistler, H. C. \& Vanetten, H. D. (1981). Phaseollin metabolism and tolerance in Fusarium solani f. sp. phaseoli. Physiological Plant Pathology 19, 257-271.

MALLETTE, M. F. (1969). Evaluation of growth in fungi by physiological and chemical means. Methods in Microbiology 1, 521-566.

Perrin, D. R. \& BotTomley, W. (1962). Studies on phytoalexins. V. The structure of pisatin from Pisum sativum L. Journal of the American Chemical Society 84, 1919-1922.

Rossall, S. \& Mansfield, J. W. (1978). The activity of wyerone acid against Botrytis. Annals of Applied Bacteriology 89, 359-362.

SkIPP, R. A. \& BAILEY, J. A. (1977). The fungitoxicity of isoflavanoid phytoalexins measured using different types of bioassay. Physiological Plant Pathology 11, 101-112.

Skipp, R. A. \& CARTer, G. A. (1978). Adaptation of fungi to isoflavanoid phytoalexins. Annals of Applied Biology 89, 366-369.

SMITH, D. A. (1976). Some effects of the phytoalexin, kievitone, on the vegetative growth of Aphanomyces euteiches, Rhizoctonia solani and Fusarium solani $\mathrm{f}$. sp. phaseoli. Physiological Plant Pathology 9, 45-55.

SPRANG, M. L. \& VANETTEN, H. D. (1978). Effects of pisatin on Fusarium solani f. sp. pisi and f. sp. cucurbitae. Phytopathology News 12, 131 (abstract).

Tegtmeier, K. J. \& VANETTEN, H. D. (1982). The role of pisatin tolerance and degradation in the virulence of Nectria haematococca on peas: a genetic analysis. Phytopathology 72, 608-612.

TrincI, A. P. J. (1972). Culture turbidity as a measure of mould growth. Transactions of the British Mycological Society 58, 467-473.

VANETTEN, H. D. (1973). Differential sensitivity of fungi to pisatin and to phaseollin. Phytopathology 63, 1477-1482.

VANETTEN, H. D. \& BARz, W. (1981). Expression of pisatin demethylating ability in Nectria haematococca. Archives of Microbiology 129, 56-60.

VAnEtTen, H. D. \& Stein, J. I. (1978). Differential response of Fusarium solani isolates to pisatin and phaseollin. Phytopathology 68, 1276-1283.

VanEtten, H. D., Pueppke, S. G. \& Kelsey, T. C. (1975). 3,6a-Dihydroxy-8,9-methylenedioxypterocarpan as a metabolite of pisatin produced by Fusarium solani f. sp. pisi. Phytochemistry 14, 1103-1105.

VanEtten, H. D., Matthews, P. S., Tegtmeier, K. J., DieterT, M. F. \& Stein, J. I. (1980). The association of pisatin tolerance and demethylation with virulence on pea in Nectria haematococca. Physiological Plant Pathology 16, 257-268.

Vanetten, H. D., Matthews, D. E. \& Smith, D. A. (1982). Metabolism of phytoalexins. In Phytoalexins, pp. 181-217. Edited by J. A. Bailey \& J. W. Mansfield. Glasgow \& London: Blackie.

Wit-Elshove, A. DE \& Fuchs, A. (1971). The infuence of the carbohydrate source on pisatin breakdown by fungi pathogenic to pea (Pisum sativum). Physiological Plant Pathology 1, 17-24. 